\title{
BMJ Open AspiriN To Inhibit SEPSIS (ANTISEPSIS) randomised controlled trial protocol
}

\author{
Damon P Eisen, ${ }^{1,2,3}$ Elizabeth M Moore ${ }^{3}$ Karin Leder, ${ }^{3,4}$ Jessica Lockery, ${ }^{3}$ \\ Emma S McBryde, ${ }^{5}$ John J McNeil, ${ }^{3}$ David Pilcher, ${ }^{3,6,7}$ Rory Wolfe, ${ }^{3}$ \\ Robyn L Woods ${ }^{3}$
}

To cite: Eisen DP, Moore EM, Leder K, et al. AspiriN To Inhibit SEPSIS (ANTISEPSIS) randomised controlled trial protocol. BMJ Open 2017;7: e013636. doi:10.1136/ bmjopen-2016-013636

- Prepublication history for this paper is available online. To view these files please visit the journal online (http://dx.doi.org/10.1136/ bmjopen-2016-013636).

Received 27 July 2016 Revised 25 November 2016 Accepted 20 December 2016

CrossMark

For numbered affiliations see end of article.

\section{Correspondence to} Dr Damon P Eisen; damon.eisen@health.qld.gov. au

\section{ABSTRACT}

Introduction: Sepsis is a leading global cause of morbidity and mortality, and is more common at the extremes of age. Moreover, the cost of in-hospital care for elderly patients with sepsis is significant. There are indications from experimental and observational studies that aspirin may reduce inflammation associated with infection. This paper describes the rationale and design of the AspiriN To Inhibit SEPSIS (ANTISEPSIS) trial, a substudy of ASPirin in Reducing Events in the Elderly (ASPREE). ANTISEPSIS primarily aims to determine whether low-dose aspirin reduces sepsis-related deaths in older people. Additionally, it will assess whether low-dose aspirin reduces sepsisrelated hospitalisations and sepsis-related Intensive Care Unit (ICU) admissions.

Methods and analysis: ASPREE is a double-blinded, randomised, placebo-controlled primary prevention trial that will determine whether daily low-dose aspirin extends disability-free longevity in 19000 healthy older people recruited in Australia and the USA. The ANTISEPSIS substudy involves additional ASPREE trial data collection to assess the impact of daily low-dose aspirin on sepsis-related events in the 16703 ASPREE participants aged 70 years and over, recruited in Australia. The intervention is a daily $100 \mathrm{mg}$ dose of enteric-coated aspirin versus matching placebo, with 1:1 randomisation. The primary outcome for the ANTISEPSIS substudy is the incidence of sepsisrelated death in eligible patients. The incidence of sepsis-related hospital and ICU admissions are secondary outcomes. ANTISEPSIS is to be conducted between 2012 and 2018.

Discussion: This substudy will determine whether aspirin, an inexpensive and accessible therapy, safely reduces sepsis-related deaths and hospitalisations in older Australians. If shown to be the case, this would have profound effects on the health of older Australians.

Trial registration number: Pre-results, ACTRN12613000349741.

\section{INTRODUCTION}

Joseph Lister pioneered a crucial aspect of modern health practice when he first used

\section{Strengths and limitations of this study}

- This trial uses the unequivocal measure of death due to sepsis as its primary end point.

- This large-scale primary prevention trial will be adequately powered to test the study hypothesis.

- There is, however, no opportunity to examine the biological basis for any demonstrated effect, as real-time samples are not available at the time of sepsis episodes.

carbolic acid solution to prevent severe infection in a contaminated wound. Antisepsis is used in all aspects of healthcare and daily life to protect us from pathogenic microorganisms. With the AspiriN To Inhibit SEPSIS (ANTISEPSIS) trial, we aim to confirm whether a simple and cheap health intervention can protect against manifestations of severe infection by modulating the human host response to infection, regardless of the specific causative microbe.

Sepsis is a lethal condition that kills one person a minute globally. Lower respiratory tract infections alone caused $5.5 \%$ of all deaths in 2012, making them the third most common cause of death. ${ }^{1}$ More specifically, in relation to our study, lower respiratory tract infections are the cause of an extremely high number of sepsis deaths in the elderly in the developed world. ${ }^{2}$

Septic shock has hospital mortality in excess of $40 \% .^{3}$ Incremental reduction in sepsis mortality has been achieved in highincome countries through early recognition and optimisation of immediate treatment delivered in well-resourced hospitals. ${ }^{4}$ However, this reduction mainly parallels general improvements in mortality of patients admitted to the ICU.

Sepsis therapy research is bedevilled with failed attempts at proving efficacy of specific treatments. ${ }^{5}$ Adjuvant treatments for sepsis 
that have been developed and trialled at major cost have failed to deliver significant reduction in mortality. There is no effective sepsis prevention strategy aimed at modulating the deleterious host inflammatory response to severe infection.

Aspirin is one of the most widely used drugs in the world today, shaping the face of modern health with its potent preventive activity against atherothrombotic vascular disease. ${ }^{6}$ Low doses of aspirin of $<150 \mathrm{mg}$ /day are sufficient for the antiplatelet effect required for stroke and cardioprotection. These low doses of aspirin are now also being recognised as mediating antiinflammatory effects, and therefore may be effective in preventing severe manifestations of sepsis. ${ }^{7}$

The cause of death in elderly patients with sepsis is usually multifactorial. Crucially though, sepsis constitutes a triggering and contributory factor for mortality. In this regard, considering multiple causes of death gives a more appropriate assessment of contributing factors to death in the elderly rather than the assessment of a single cause of death. Adding a simple preventive therapy with overlapping effects in reduction of cardiovascular and sepsis risks would be of major benefit to the health of Australia's ageing population by reducing health costs. Aspirin has the potential to fulfil that role.

Examination of the biological bases of aspirin in limiting the deleterious effects of the septic inflammatory cascade is illuminating. Aspirin has effects on at least three sepsis/inflammation pathways.

Tumour necrosis factor (TNF) and interleukin-6 are canonical proinflammatory cytokines. In sepsis, immune cell receptors recognise pathogen associated molecular patterns mediating intracellular signalling events that result in nuclear factor kappa-beta (NFкB) activation. This then results in transcription of TNF. NFאB activation is inhibited by aspirin and other non-steroidal antiinflammatory drugs (NSAIDs). This is mediated by inhibition of Ikk-B. ${ }^{8}$ The concentration of NSAIDs required for this inhibition has been measured to be lower than aspirin. ${ }^{9}$ The low range of aspirin for this effect has not been defined.

Lipid mediators of sepsis have recently been described. ${ }^{10}$ A number of these molecules act as antiinflammatories and also to restore homeostasis. ${ }^{11}{ }^{12}$ In sepsis, they reduce established inflammation by mechanisms including restoration of polymorphonuclear apoptosis which limits continued production of proinflammatory cytokines in tissues and increase of nitric oxide synthesis. ${ }^{13}$ Low doses of aspirin have been shown to increase lipoxins (aspirin triggered lipoxin (ATL)) and resolvins in vitro ${ }^{11} 12$ and in human trials. ${ }^{14}$ Furthermore, ATL mediated, salutary effects have been shown in animal and human disease models of sepsis and inflammation. Animal models demonstrate that aspirin given both before and after onset of sepsis reduces mortality. ${ }^{15}$ Low-dose aspirin increases nitric oxide production as seen in experimental animals. ${ }^{16}$ Additionally, in a human model, ATL accounts for prevention of skin blister via reduced neutrophil and macrophage accumulation. ${ }^{17}$ Crucially, these beneficial effects are unique to aspirin among the NSAIDs as it alone increases ATL.

Platelets become activated in sepsis due to interaction with invading bacteria via three broad mechanisms. These constitute: (1) binding of bacteria to plasma proteins which are ligands for platelet receptors, (2) direct bacterial binding to platelet receptors and (3) secretion of aspirin binding bacterial products such as toxins. ${ }^{18}$ Aspirin reduces activation of platelets by inhibition of cyclooxygenase $\mathrm{I}^{19}$

Bleeding risks in patients taking regular aspirin are clearly defined. Among primary prevention trial participants taking between 700 and $1625 \mathrm{mg}$ aspirin per week, the increased risk of all gastrointestinal haemorrhage was $1.27(95 \%$ CI 1.05 to 1.55$) .{ }^{20}{ }^{21}$ This must be balanced against potential benefits of low-dose aspirin therapy, including for sepsis prevention.

Numerous observational studies have investigated the influence of long-term aspirin use prior to onset of sepsis. ${ }^{22-34}$ A number of these propensity-matched studies show that long-term aspirin use is associated with reduced mortality. ${ }^{22-28} 30-32$

These observational data, along with the effect of low-dose aspirin on inflammatory pathways, provide a compelling background for investigation of possible reduction in sepsis deaths in ANTISEPSIS. Therefore, this doubled blind, placebo controlled, randomised controlled primary prevention trial (RCT) will investigate whether low-dose aspirin reduces deaths due to sepsis.

\section{STUDY METHODS}

ANTISEPSIS is a substudy of the ASPirin in Reducing Events in the Elderly study (ASPREE). ASPREE is an Australian/US randomised, double blind, placebo controlled, primary prevention trial of low-dose aspirin in the elderly. ASPREE will measure the effect of low-dose aspirin on numerous outcomes: all-cause mortality, incident dementia, persistent physical disability, cardiovascular and major haemorrhagic events, and cancer incidence. ANTISEPSIS is embedded in the study design of the ASPREE principal study. Monitoring and event reporting for ANTISEPSIS is conducted with NHMRC funding of staff within the ASPREE data and document collection team. ANTISEPSIS is being conducted between 2012 and 2018.

\section{Hypothesis and aims}

We hypothesise that severe outcomes relating to sepsis in the elderly may be prevented by daily low-dose aspirin. We will conduct the ANTISEPSIS trial using the infrastructure of the ASPREE RCT. We will extend sepsis event data collection in ASPREE participants to assess our primary end point:

- Reduction of deaths contributed to by sepsis in participants receiving aspirin versus placebo. 
We will also conduct an analysis of two secondary end points:

- Reduction of sepsis episodes requiring hospital admissions

- Reduction of intensive care unit (ICU) admissions among patients hospitalised for severe sepsis.

\section{Antisepsis study design}

Detailed methods for the ASPREE 'principal' study are described in the ASPREE protocol available on the aspree.org website-http://www.aspree.org/AUS/aspreecontent/aspree-study- details/aspree-materials.aspx/ and in the ASPREE methods paper. ${ }^{35}$

Only the Australian ASPREE participants are included in the ANTISEPSIS study. This stems from the adequacy of the Australian ASPREE sample size for the ANTISEPSIS end points. Furthermore, the absence of detailed ICU data on participants from the USA means that there would be insufficient data to adjudicate all sepsis end points.

The design of the ANTISEPSIS trial mirrors that of the principal ASPREE study. ANTISEPSIS is also a trial of $100 \mathrm{mg}$ enteric-coated aspirin taken daily versus matching placebo. ASPREE trial participants were recruited through their primary care general practices. One to one randomisation of Australian participants was stratified for general practice and for age (70-79 or $\geq 80$ years).

ANTISEPSIS recruits from ASPREE participants and hence the exclusion/inclusion criteria for the ASPREE study apply. The participants in the ASPREE principal trial were required to be free of previous cardiovascular disease or stroke, have preserved intellectual function and have no known illness that would preclude their follow-up participation within the next 3-5 years. Apart from this, they are broadly representative of the healthy elderly population.

The details on: study medication and supplies, screening visit and run-in placebo for the ASPREE trial, randomisation visit, scheduled visits and measurements are as per the ASPREE protocol. ${ }^{35}$

\section{Antisepsis end point determination}

Demographic data describing age, sex, body habitus, comorbidities, smoking history, alcohol intake and concomitant medications at ASPREE entry are available from the ASPREE study. Data related to the three end points of the ANTISEPSIS trial are available from general practitioner (GP) clinics, hospital documentation and death certificates. Additional demographic, severity of illness and outcome data on patients admitted to ICUs will be obtained by merging ASPREE data with information from the Australian and New Zealand Intensive Care Society (ANZICS) Adult Patient Database (APD), one of four clinical quality registries run by the ANZICS Centre for Outcome and Resource Evaluation (CORE).

Since ASPREE data only provide International Classification of Diseases 10th Revision hospital primary admission codes, ANTISEPSIS will examine all hospital discharge summaries and death certificates from ASPREE participants identified as having a possible sepsis end point to ensure complete capture of sepsis episodes. The search for hospitalisation data relies on a number of source materials. The ASPREE operational process captures all hospitalisations, although some hospital discharge summaries may be blank. In that instance, GP records will be examined for information regarding the reason for hospitalisation. ASPREE participants also report admission(s) on yearly review and ASPREE operational processes also identify participant deaths. Death Certificates are retrieved for ASPREE patients who die outside of an acute hospital admission. Missing data on hospitalisation admission/discharge diagnoses are flagged for priority retrieval by ASPREE monitors/study personnel at GP visits.

ANTISEPSIS study research staff extract information relating to:

- Nature of sepsis episode, diagnosis, infecting organism (if available).

- Sepsis severity; ICU admission, disease severity at ICU admission (Acute Physiology Chronic Health Evaluation (APACHE) II score), prognostic score (APACHE risk of death (APACHE II score with adjustment for site of infection))

- Sepsis episode outcome; survival or death.

- Time of sepsis episode from ASPREE study medication initiation for survival analysis.

Data extracted from the ANZICS APD include:

- Sepsis severity and criteria, ICU length of stay, disease severity at ICU admission (APACHE II and III scores), predicted risk of death (derived from the APACHE III scoring system and using the 'ANZ Risk of Death' model), ICU and hospital outcomes.

An electronic case report form has been designed for input of ANTISEPSIS Study end point data into the ASPREE web-based data acquisition system.

\section{Antisepsis case definitions \\ Sepsis definition}

Sepsis is defined as the presence of the systemic inflammatory response syndrome (SIRS; the presence of two or more of: temperature $<36^{\circ} \mathrm{C}$ or $>38^{\circ} \mathrm{C}$, heart rate $>90 \mathrm{bpm}$, respiratory rate $>20$ breaths $/$ min or $\mathrm{PaCO}_{2}$ $<32 \mathrm{~mm} \mathrm{Hg}$, and white cell count $>12000$ or $<4000$ cells $/ \mathrm{mm}^{3}$ or $>10 \%$ bands) ${ }^{36}$ plus documented infection as described below. The use of SIRS criteria for definition of sepsis relates to the timing of ANTISEPSIS protocol development and study initiation. ANTISEPSIS started before the development of the Third International Consensus Definitions for Sepsis and Septic Shock (Sepsis-3). ${ }^{3}$

\section{Infection site definitions}

Primary blood stream infection is defined as recognised pathogen (defined as a microorganism not usually regarded as a common skin contaminant) cultured from 
one or more blood cultures, or a common skin contaminant cultured from two or more blood cultures drawn on separate occasions and the organism cultured from blood is not related to an infection at another site, including intravascular-access devices. ${ }^{37}$

Pneumonia is defined as a chest radiograph within 24 hours of hospital admission demonstrating features consistent with acute pneumonia; and at least two symptoms consistent with pneumonia (eg, fever or hypothermia, rigours, sweats, new cough (with or without sputum), chest discomfort or new onset of dyspnoea). ${ }^{38}$

Hospital-acquired pneumonia is defined as pneumonia that occurs 48 hours or more after admission, which was not incubating at the time of admission. ${ }^{39}$

Meningitis is defined as increased white cells, elevated protein and decreased glucose in cerebrospinal fluid (CSF) (per reporting laboratory's reference range), or organisms seen on Gram stain of CSF, or organisms identified from blood by a culture or non-culture-based microbiological testing method which is performed for purposes of clinical diagnosis or treatment. ${ }^{40}$

Urinary tract infection (UTI) is defined as at least one of: fever $>38^{\circ} \mathrm{C}$, suprapubic tenderness, costovertebral angle pain or tenderness, urinary frequency, urinary urgency or dysuria and urine culture with no more than two species of organisms, at least one of which is a bacterium of $\geq 10^{5}$ colony forming units $/ \mathrm{mL}^{41}$ UTI is either non-catheter associated or, where a urinary catheter has been in situ for at least 2 days, catheter associated.

\section{Skin and soft tissue infection:}

A. Cellulitis is defined as any spreading infection involving the dermis and subcutaneous tissues. ${ }^{42}$

B. Abscess is defined as any collection of pus within the dermis or subcutaneous tissues. ${ }^{42}$

C. Necrotising soft tissue infection is defined as a necrotising infection involving any of the soft tissue layers, including the dermis, subcutaneous tissue, superficial or deep fascia, and muscle. ${ }^{42}$

Peritonitis is defined as a clinically compatible illness with abdominal pain and guarding associated with documented evidence of perforation (free air in the abdomen on radiographic studies or surgical confirmation of peritoneal inflammation following luminal perforation). ${ }^{37}$

Bone and joint infection is defined as the presence of organisms grown from bone or joint tissues by microbiological culture in a patient with bony pain, tenderness or drainage. ${ }^{40}$

Infective endocarditis is diagnosed according to modified Duke's Criteria. ${ }^{43}$

Gastroenteritis is defined as the acute onset of diarrhoea (liquid stools for $>12$ hours) and no likely non-infectious cause with an enteric pathogen identified from stool or rectal swab by a culture or non-culture-based microbiological method. ${ }^{40}$

The approval by the Monash University Human Research Ethics Committee for ANTISEPSIS includes authorisation to contact local pathology services to extract individual patient pathology results to confirm the presence of sepsis.

\section{End point definitions}

The primary end point, death due to sepsis, is defined as death of an ASPREE participant who had been either admitted to hospital for an infection episode or where such an episode of infection develops in hospital and in either case, the infection episode contributes to death (as determined by hospital records and/or a death certificate). If ASPREE participants die out of hospital, the death certificate will be used to determine whether sepsis was a contributory cause of death.

Secondary study end points are defined as (1) nonfatal sepsis during hospital admission of an ASPREE participant due to an infection episode defined as above, (2) non-fatal sepsis/septic shock during ICU admission of an ASPREE participant due to an infection episode defined as above.

\section{End point adjudication}

An end point adjudication committee consisting of ANTISEPSIS investigators who are Infectious Diseases and ICU staff specialist physicians decides outcomes. The adjudication process is web-based and all those involved are blinded to group allocation. Two ANTISEPSIS adjudicators review each event independently. Where there is agreement, this is the outcome of the event. Discordant results go to a third reviewer for the final adjudication.

\section{Trial safe conduct-data safety monitoring board}

The safety routine for ANTISEPSIS is as established for ASPREE. All serious adverse events and adverse events are registered with the ASPREE trial according to the established protocol and then presented to an independent Data Safety and Monitoring Board (DSMB; established by the National Institute on Ageing) and to Human Research Ethics Committees. The DSMB oversees the ASPREE study to monitor quality control of the data, progress of recruitment and safety aspects of the ASPREE trial.

\section{Sample size and study power}

At the time that ANTISEPSIS was designed, available observational data showed a relative effect size on sepsis mortality in patients in ICU taking long-term aspirin prior to ICU admission, in the order of $40-80 \% .^{22} 25$ These data are from two studies with a fundamentally different design. One was an observational study of Australian patients in ICU, which showed that patients taking aspirin prior to hospitalisation with SIRS who were continued on aspirin had an OR of 0.6 for in-hospital mortality compared with those not treated with aspirin. The HR calculated from this study for mortality in patients with SIRS treated with aspirin was 0.63. The group of patients with proven sepsis had an OR of 0.52 for mortality. ${ }^{22}$ The other single centre German 
study of aspirin in ICU patients showed reduced mortality (OR 0.19) in patients with an unrestricted range of admission diagnoses. ${ }^{25}$

Using an HR of 0.63 to examine the sample size required to power our ANTISEPSIS study for the outcome of sepsis-related mortality is a conservative approach given the lower ORs available from the German study and Australian patients with proven sepsis. However, we reasoned that the SIRS OR was the most reliable representation of the likely magnitude of aspirin effects on the inflammatory pathways common to SIRS and sepsis given that this figure is based on a large cohort $(n=5525)$ of first SIRS episodes.

The ASPREE study rate of death was estimated to be 17.6 per 1000 participant years. Approximately 4.75 years average follow-up time per participant will be available in an analysis of time to death. We assumed that $20 \%$ of the deaths in ASPREE participants would be contributed to by sepsis (as supported by Australian data $^{30}$ ). This provides an event rate for the primary end point of ANTISEPSIS of 3.5 per 1000 participant years. Anticipating half of 16000 Australian ASPREE participants to be randomised to placebo and an event rate of 3.5 per 1000 participant year, we expect 133 primary end point events in the placebo group. With this number of sepsis-related deaths, we would have $80 \%$ power to detect an HR of 0.69 for aspirin versus placebo groups. If only $15 \%$ of ASPREE deaths are contributed to by sepsis, we would instead expect 100 primary end point events in the control group and still have $80 \%$ power to detect an HR of 0.65 . In either of these scenarios, the ANTISEPSIS study is powered to detect an effect less than the HR of 0.63 measured previously. ${ }^{26}$ The two secondary end points, admission to hospital for sepsis and admission to ICU for sepsis, are more common than death due to sepsis, ${ }^{3}{ }^{4}$ so they will be powered to detect smaller effects than outlined above for the primary end point.

\section{Data analysis}

We will analyse our primary and two secondary end points without Bonferroni correction using univariate survival analysis methods: the log-rank test and Cox proportional hazards regression. The proportional hazards assumption will be tested as part of the analysis. In subsequent analysis, we will adjust for the following variables known to influence mortality due to sepsis: diabetes, age at recruitment, malignancy, alcohol intake and smoking or chronic lung disease.

The main analyses will compare participant groups as randomised, that is, intention to treat. A per protocol analysis will also be performed.

ANTISEPSIS trial results will be reported according to the CONSORT statement. ${ }^{44}$

\section{Ethical conduct of the trial}

This study will be conducted in accordance with the ICH GCP Note for Guidance on Good Clinical Practice
(CPMP/ICH/135/95) annotated with TGA comments and NH\&MRC National Statement on Ethical Conduct in Human Research 2007 protocols, and in keeping with local regulations.

\section{Informed consent}

The need for individual informed consent for inclusion of ASPREE participants in ANTISEPSIS has been waived by the Monash University Human Research Ethics Committee in its approval of the ANTISEPSIS study. The ANTISEPSIS study conforms to the conditions set out in CHAPTER 2.3: QUALIFYING OR WAIVING CONDITIONS FOR CONSENT in the National Statement on Ethical Conduct in Human Research 2007.

\section{Trial registration}

ANTISEPSIS is registered with the Australian New Zealand Clinical Trials Registry ACTRN12613000349741. The ASPREE study is registered with the International Standard Randomised Controlled Trials Register, ASPirin in Reducing Events in the Elderly, ISRCTN83772183 and clinicaltrials.gov NCT01038583.

\section{DISCUSSION}

Primary prevention with aspirin appears to reduce allcause mortality. A meta-analysis of 100000 aspirin primary prevention trial patient outcomes showed that this reduced all-cause mortality (OR, 0.94; 95\% CI 0.88 to 1.00 ) and that this reduction was due neither to reduced cardiovascular nor cancer deaths. ${ }^{21}$ However, deaths due to cancer do appear to be reduced in a meta-analysis of long-term follow-up of 25570 participants from primary and secondary prevention aspirin trials. Here, the reduction of cancer deaths with aspirin therapy increased with duration of treatment. ${ }^{45}$ This finding can therefore be viewed as being separate from the non-cancer reduction of all-cause mortality from primary prevention studies. No analysis of noncardiovascular or non-cancer mortality has been undertaken on data from primary or secondary aspirin prevention studies. It may be that one of the previously unmeasured pathways by which aspirin reduces all-cause mortality is via a reduction in sepsis deaths when aspirin is used for primary prevention. We will test this hypothesis in the ANTISEPSIS trial.

Recruitment to ASPREE was completed in December 2014 with a final number of 16703 participants in Australia. The collection of information on end points will conclude at the end of 2017 and the unblinding of the principal ASPREE trial, and therefore ANTISEPSIS, is expected in mid-2018. If aspirin is shown to be effective in reducing the impact of severe sepsis, it will be the first time that such an outcome has been demonstrated.

\section{Author affiliations}

${ }^{1}$ Townsville Hospital and Health Service, Townsville, Queensland, Australia ${ }^{2}$ Division of Tropical Health and Medicine, College of Medicine and Dentistry, James Cook University, Townsville, Queensland, Australia 
${ }^{3}$ Department of Epidemiology and Preventive Medicine, Monash University, Melbourne, Victoria, Australia

${ }^{4}$ Victorian Infectious Diseases Service at the Peter Doherty Institute, Royal Melbourne Hospital, Melbourne, Victoria, Australia

${ }^{5}$ Division of Tropical Health and Medicine, Australian Institute of Tropical Health and Medicine, James Cook University, Townsville, Queensland, Australia

${ }^{6}$ Department of Intensive Care, The Alfred Hospital, Melbourne, Victoria, Australia

${ }^{7}$ The Australian and New Zealand Intensive Care Society (ANZICS) Centre for Outcome and Resource Evaluation (CORE), Melbourne, Victoria, Australia

Acknowledgements The investigators acknowledge the National Institute on Ageing (NIA; number R01-AG029824) for funding the principal ASPREE trial in Australia and the USA, and the National Health \& Medical Research Council (grant number 334047), the Victorian Cancer Agency and Monash University for funding support of ASPREE in Australia. The investigators also acknowledge the work of all ASPREE field research staff, and those within the ASPREE data team who source the supporting documents from hospitals and pathology services. The investigators would particularly like to acknowledge the valued contribution of the ASPREE participants and the support from their general practitioners.

Contributors DPE conceived the study. DPE, KL, ESMcB, DP, RW and RLW wrote the study protocol. JL designed the database used in the study. JJM is responsible for the ASPREE study on which this study protocol is based. $E S M c B$ and RLW designed the statistical analysis plan for the protocol. DPE, $\mathrm{KL}, \mathrm{ESMcB}, \mathrm{DP}, \mathrm{RW}$ and $\mathrm{EM}$ designed the implementation aspects of this protocol. All authors critically reviewed the manuscript.

Funding The ANTISEPSIS Study is funded by The National Health \& Medical Research Council project grant number 1041986 (2013-2017).

Competing interests None declared.

Ethics approval Monash University Human Research Ethics Committee approved the ANTISEPSIS study (CF13/466-2013000204, 9 April 2013).

Provenance and peer review Not commissioned; externally peer reviewed.

Data sharing statement Only aggregate data will be available in a results publication.

Open Access This is an Open Access article distributed in accordance with the Creative Commons Attribution Non Commercial (CC BY-NC 4.0) license, which permits others to distribute, remix, adapt, build upon this work noncommercially, and license their derivative works on different terms, provided the original work is properly cited and the use is non-commercial. See: http:// creativecommons.org/licenses/by-nc/4.0/

\section{REFERENCES}

1. World Health Organisation. The top 10 causes of death. 2014. http:/ www.who.int/mediacentre/factsheets/fs310/en/index.html

2. Global Burden of Diseases Study, Mortality, Causes of Death Committee. Global, regional, and national age-sex specific all-cause and cause-specific mortality for 240 causes of death, 1990-2013: a systematic analysis for the Global Burden of Disease Study 2013. Lancet 2015;385:117-71.

3. Singer M, Deutschman CS, Seymour CW, et al. The Third International Consensus Definitions for Sepsis and Septic Shock (Sepsis-3). JAMA 2016;315:801-10.

4. Kaukonen KM, Bailey M, Suzuki S, et al. Mortality related to severe sepsis and septic shock among critically ill patients in Australia and New Zealand, 2000-2012. JAMA 2014;311:1308-16.

5. Cohen J, Vincent JL, Adhikari NK, et al. Sepsis: a roadmap for future research. Lancet Infect Dis 2015;15:581-614.

6. Goodman SG, Menon V, Cannon CP, et al. Acute ST segment elevation myocardial infarction: American College of Chest Physicians Evidence-Based Clinical Practice Guidelines (8th Edition). Chest 2008;133(Suppl 6):708S-75S.

7. Eisen DP. Manifold beneficial effects of acetyl salicylic acid and nonsteroidal anti-inflammatory drugs on sepsis. Intensive Care Med 2012;38:1249-57.

8. Kopp E, Ghosh S. Inhibition of NF-kappa B by sodium salicylate and aspirin. Science 1994;265:956-9.
9. Takada Y, Bhardwaj A, Potdar P, et al. Nonsteroidal anti-inflammatory agents differ in their ability to suppress NF-kappaB activation, inhibition of expression of cyclooxygenase-2 and cyclin D1, and abrogation of tumor cell proliferation. Oncogene 2004;23:9247-58.

10. Serhan CN, Haeggström JZ, Leslie CC. Lipid mediator networks in cell signaling: update and impact of cytokines. FASEB $J$ 1996;10:1147-58

11. Serhan CN, Chiang N. Lipid-derived mediators in endogenous anti-inflammation and resolution: lipoxins and aspirin-triggered 15-epi-lipoxins. ScientificWorldJournal 2002;2:169-204.

12. Arita M, Yoshida M, Hong S, et al. Resolvin E1, an endogenous lipid mediator derived from omega-3 eicosapentaenoic acid, protects against 2,4,6-trinitrobenzene sulfonic acid-induced colitis. Proc Natl Acad Sci USA 2005;102:7671-6.

13. Paul-Clark MJ, Van Cao T, Moradi-Bidhendi N, et al. 15-epi-lipoxin A4-mediated induction of nitric oxide explains how aspirin inhibits acute inflammation. J Exp Med 2004;200:69-78.

14. Chiang N, Bermudez EA, Ridker PM, et al. Aspirin triggers antiinflammatory 15-epi-lipoxin A4 and inhibits thromboxane in a randomized human trial. Proc Natl Acad Sci USA 2004;101:15178-83.

15. El Kebir D, József L, Pan W, et al. 15-epi-lipoxin A4 inhibits myeloperoxidase signaling and enhances resolution of acute lung injury. Am J Respir Crit Care Med 2009;180:311-19.

16. Hennekens $\mathrm{CH}$, Schneider WR, Pokov $\mathrm{A}$, et al. A randomized trial of aspirin at clinically relevant doses and nitric oxide formation in humans. J Cardiovasc Pharmacol Ther 2010;15:344-8.

17. Morris T, Stables M, Hobbs A, et al. Effects of low-dose aspirin on acute inflammatory responses in humans. J Immunol 2009;183:2089-96.

18. Cox D, Kerrigan SW, Watson SP. Platelets and the innate immune system: mechanisms of bacterial-induced platelet activation. $J$ Thromb Haemost 2011;9:1097-107.

19. Masotti G, Galanti G, Poggesi L, et al. Differential inhibition of prostacyclin production and platelet aggregation by aspirin in humans. Adv Prostaglandin Thromboxane Res 1980;6:317-20.

20. Huang ES, Strate LL, Ho WW, et al. Long-term use of aspirin and the risk of gastrointestinal bleeding. Am J Med 2011;124:426-33.

21. Seshasai SR, Wijesuriya S, Sivakumaran R, et al. Effect of aspirin on vascular and nonvascular outcomes: meta-analysis of randomized controlled trials. Arch Intern Med 2012;172:209-16.

22. Eisen DP, Reid D, McBryde ES. Acetyl salicylic acid usage and mortality in critically ill patients with the systemic inflammatory response syndrome and sepsis. Crit Care Med 2012;40:1761-7.

23. Lösche W, Boettel J, Kabisch B, et al. Do aspirin and other antiplatelet drugs reduce the mortality in critically ill patients? Thrombosis 2011;2012:720254.

24. Winning J, Neumann J, Kohl M, et al. Antiplatelet drugs and outcome in mixed admissions to an intensive care unit. Crit Care Med 2010;38:32-7.

25. Winning J, Reichel J, Eisenhut Y, et al. Anti-platelet drugs and outcome in severe infection: clinical impact and underlying mechanisms. Platelets 2009;20:50-7.

26. Chen W, Janz DR, Bastarache JA, et al. Prehospital aspirin use is associated with reduced risk of acute respiratory distress syndrome in critically ill patients: a propensity-adjusted analysis. Crit Care Med 2015;43:801-7.

27. Otto GP, Sossdorf M, Boettel J, et al. Effects of low-dose acetylsalicylic acid and atherosclerotic vascular diseases on the outcome in patients with severe sepsis or septic shock. Platelets 2013;24:480-5

28. Valerio-Rojas JC, Jaffer IJ, Kor DJ, et al. Outcomes of severe sepsis and septic shock patients on chronic antiplatelet treatment: a historical cohort study. Crit Care Res Pract 2013;2013:782573.

29. Boyle AJ, Di Gangi S, Hamid UI, et al. Aspirin therapy in patients with acute respiratory distress syndrome (ARDS) is associated with reduced intensive care unit mortality: a prospective analysis. Crit Care 2015;19:109.

30. Falcone M, Russo A, Cangemi R, et al. Lower mortality rate in elderly patients with community-onset pneumonia on treatment with aspirin. J Am Heart Assoc 2015;4:e001595.

31. Sossdorf M, Otto GP, Boettel J, et al. Benefit of low-dose aspirin and non-steroidal anti-inflammatory drugs in septic patients. Crit Care 2013;17:402.

32. Tsai MJ, Ou SM, Shih CJ, et al. Association of prior antiplatelet agents with mortality in sepsis patients: a nationwide population-based cohort study. Intensive Care Med 2015;41:806-13.

33. Al Harbi SA, Tamim HM, Al-Dorzi HM, et al. Association between aspirin therapy and the outcome in critically ill patients: a nested cohort study. BMC Pharmacol Toxicol 2016;17:5. 
34. Wiewel MA, de Stoppelaar SF, van Vught LA, et al. Chronic antiplatelet therapy is not associated with alterations in the presentation, outcome, or host response biomarkers during sepsis: a propensity-matched analysis. Intensive Care Med 2016;42:352-60.

35. ASPREE Investigators Group. Study design of ASPirin in Reducing Events in the Elderly (ASPREE): a randomized, controlled trial. Contemp Clin Trials 2013;36:555-64.

36. Bone RC, Balk RA, Cerra FB, et al. Definitions for sepsis and organ failure and guidelines for the use of innovative therapies in sepsis. The ACCP/SCCM Consensus Conference Committee. American College of Chest Physicians/Society of Critical Care Medicine. Chest 1992;101:1644-55.

37. Calandra T, Cohen J. International Sepsis Forum Definition of Infection in the ICUCC. The international sepsis forum consensus conference on definitions of infection in the intensive care unit. Crit Care Med 2005;33:1538-48.

38. Mandell LA, Wunderink RG, Anzueto A, et al. Infectious Diseases Society of America/American Thoracic Society consensus guidelines on the management of community-acquired pneumonia in adults. Clin Infect Dis 2007;44(Suppl 2):S27-72.

39. Tablan OC, Anderson LJ, Besser R, et al. Guidelines for preventing healthcare-associated pneumonia, 2003: recommendations of $\mathrm{CDC}$ and the Healthcare Infection Control Practices Advisory Committee. MMWR Recomm Rep 2004;53:1-36.
40. Centers for Disease Control. CDC/NHSN Surveillance Definitions for Specific Types of Infections. Secondary CDC/NHSN Surveillance Definitions for Specific Types of Infections. 2016. http://www.cdc. gov/nhsn/PDFs/pscManual/17pscNosInfDef_current.pdf

41. Centers for Disease Control. Urinary Tract Infection (Catheter-Associated Urinary Tract Infection [CAUTI] and Non-Catheter-Associated Urinary Tract Infection [UTI]) and Other Urinary System Infection [USI]) Events. Secondary Urinary Tract Infection (Catheter-Associated Urinary Tract Infection [CAUTI] and Non-Catheter-Associated Urinary Tract Infection [UTI]) and Other Urinary System Infection [USI]) Events. 2016. http://www.cdc.gov/ nhsn/PDFs/pscManual/17pscNosInfDef current.pdf

42. Gunderson CG. Cellulitis: definition, etiology, and clinical features. Am J Med 2011;124:1113-22.

43. Li JS, Sexton DJ, Mick N, et al. Proposed modifications to the Duke criteria for the diagnosis of infective endocarditis. Clin Infect Dis 2000;30:633-8.

44. Moher D, Hopewell S, Schulz KF, et al. CONSORT 2010 Explanation and Elaboration: updated guidelines for reporting parallel group randomised trials. J Clin Epidemiol 2010;63: e1-37.

45. Rothwell PM, Fowkes FG, Belch JF, et al. Effect of daily aspirin on long-term risk of death due to cancer: analysis of individual patient data from randomised trials. Lancet 2011;377:31-41. 